\title{
A New and Simple Model for the Prediction of Horizontal Well Productivity in Gas Condensate Reservoirs Ghahri P. ${ }^{1,}$ and Jamiolahmady M.
}

\begin{abstract}
Horizontal wells are a proven and acknowledged technology to enhance well productivity through an increase in reservoir contact compared to that of vertical wells under the same conditions. In the last three decades, a considerable effort has been directed by many investigators to study flow around horizontal wells. In gas condensate reservoirs, in addition to the three dimensional (3D) nature of the flow geometry, the flow behaviour is further complicated by the phase change and the variation of relative permeability ( $\mathrm{kr}$ ) due to the coupling (increase in $\mathrm{k}_{\mathrm{r}}$ by an increase in velocity or decrease in IFT) and inertia (a decrease in $\mathrm{k}_{\mathrm{r}}$ by an increase in velocity) effects. Therefore, simulating such a complex 3D flow using commercial numerical simulators requires a 3D fine grid compositional approach which is very impractical, cumbersome and sometimes triggers convergence problems due to numerical instability. So far, none of these studies propose a method to deal with the complex multiphase behaviour of gas condensate flow around the horizontal well. Consequently, the introduction of a quick and reliable tool for long term productivity calculation in such a system is much needed.

This paper presents a technique which was developed through a comprehensive study of the flow behaviour around horizontal wells in gas condensate reservoirs involving the creation of many in-house mathematical models using finite element and finite difference methods. An in-house simulator was developed to accurately model the multiphase flow of gas and condensate around horizontal wells. A large data bank was then generated covering the impact of a wide range of pertinent geometric and flow parameters on well performance including: well and reservoir geometries, reservoir and bottom-hole pressure, fluid velocity, gas oil ratio and fluid composition.

Based on the results of the simulation, a new method has been proposed to predict the productivity of horizontal wells for the case of multiphase flow of gas and condensate. In this approach, the flow behaviour of gas and condensate around the well is quantified in terms of the effective wellbore radius of an equivalent open hole that replicates flow around the actual 3D system. The effective wellbore radius varies with fluid properties, velocity and interfacial tension (IFT), reservoir and wellbore conditions. The integrity of the new methodology has also been verified for various fluids and flow conditions.
\end{abstract}

With this approach, a simple spreadsheet, without recourse to complex numerical simulation, can predict the horizontal well performance, significantly facilitating engineering and management decisions on the application of costly horizontal well technologies.

Keywords: gas condensate flow; horizontal wells; effective wellbore radius; well productivity; interfacial tension

\footnotetext{
${ }^{1}$ Corresponding author. Tel.: +44-1224-272146, fax +44-1224-272497.

E-mail address: panteha.ghahri@abdn.ac.uk
} 


\section{INTRODUCTION}

In gas condensate reservoirs, as the pressure falls below the dew point pressure, a bank of condensate forms around the wellbore which affects the flow behaviour and consequently well productivity. Under such condition, two hydrocarbon phases (gas and condensate) co-exit under making the phase and flow behaviour completely different from those of dry gas reservoirs. It is also well documented that the fluid flow behaviour around the wellbore region, of near critical gas condensate systems, characterized by very low interfacial tension, is different from that of conventional oil gas systems. Thus, the relative permeability of gas condensate systems has a unique dependency on interfacial tension (Bardon and Longeron 1980, Asar and Handy 1988) and velocity (Danesh et. al, 1994, Henderson et. al 1996, Ali et. al 1997, Bloom et. al 1997). Accordingly, any reservoir simulator or model proposed for well calculations in such systems must take into account these effects in order to make a sufficiently accurate prediction of the well performance.

The reduction of relative permeability permeability at high velocities due to negative inertia (non-Darcy flow) was first introduced by Forchhiemer (1914). The dependency of the relative permeability of low IFT systems on interfacial tension was first reported by Bardon (1980). The improvement of the relative permeability of gas and condensate fluids due to an increase in velocity was first reported experimentally by the gas condensate recovery research team of Heriot-Watt University (Danesh et al. 1994, Henderson et al. 1996). The positive coupling effect, which refers to the improvement of relative permeability as velocity increases and/or IFT decreases, has been shown theoretically and experimentally to be due to the simultaneous coupled flow of the gas and condensate phases with intermittent opening and closure of the gas passages by the condensate at the pore level (Jamiolahmady et al. 2000, 2003).

The breakthrough of drilling technology in developing and completing horizontal wells has significantly impacted oil and gas reservoir development strategies. Such a well trajectory increases the well exposure to the reservoir drainage area and thereby appreciably increases the well productivity. However, the cost of horizontal wells is often a major barrier. Over the last few years, since horizontal wells have been drilled more economically, the number of horizontal wells has grown exponentially worldwide. Therefore, many studies have been conducted to propose an accurate formulation for the calculation of the productivity of such wells at steady state (SS) or pseudo steady state (PSS) conditions ((Borisov, 1964), (Joshi, 1991), (Giger et al., 1984), (Permadi, 1995), (Renard and Dupuy, 1991), (Lu, 2001), (Elgaghah et al., 1996), (Economides et al., 1996), (Furui et al., 2003), (Wang and Eaton, 2007), (Sheng and J, 2007), (Escobar and Montealegre, 2008), (Economides et al., 1996), (Helmy and Wattenbarger, 1998), (Lu and Tiab, 2007), (Babu and Odeh, 1989) and (Goode and Kuchuk, 1991)). However, these equations are only applicable for single phase flow conditions.

There are only a few sensitivity studies which have discussed horizontal well performance in gas condensate reservoirs. Numerical simulations conducted by (Hashemi and Gringarten, 2005), (Dehane and Tiab, 2000), (Marir and Djebbar, 2006) and (Jamiolahmady and Danesh, 2007) have all mainly been focused on the sensitivity of pertinent parameters on the performance of such wells.

As noted earlier, the flow behaviour of gas condensate reservoirs is very complex due to phase change, inertia and coupling. An accurate estimation of the productivity of such systems using a numerical simulator is a challenging task because this 3D compositional simulation requires a fine grid to capture the abrupt variation of fluid and flow parameters around the wellbore. This is cumbersome and impractical for field applications. 
This work is devoted to study flow behaviour around a horizontal well by developing a 3D horizontal well two phase compositional (gas condensate) mathematical simulator under steady state conditions, and proposing a model to predict the horizontal well productivity for such systems. In this work, we used a generalized correlation for $\mathrm{kr}$ calculation in the in-house simulator developed by Jamiolahmady et al. 2009. A brief description of the formulations accounting for coupling and inertial effects on $\mathrm{kr}$ is explained in Appendix A. The main advantages of this correlation compared to other formulations available in the literature are that it uses either universal parameters or those parameters that can be estimated from readily available petrophysical data and it also accounts for the combined effect of inertia and coupling.

In our proposed approach, using the results of the developed 3D horizontal well in-house simulator, an effective wellbore radius is defined for an equivalent open hole radial system replicating the two phase flow of gas and condensate around the horizontal well. As demonstrated here, this formulation and methodology appropriately correlate the impact of pertinent parameters including IFT, velocity, phase changes and the well geometries parameters, i.e. wellbore radius, reservoir thickness and horizontal well length, to the results of the exact solution obtained by the corresponding in-house simulator operating under the same flowing conditions. The proposed formulation, which is simple and easy to use, correctly describes single phase non-Darcy (inertial) flow systems when total gas fractional flow is unity and if velocity is low to Darcy flow conditions.

\section{FLOW SIMULATION}

In this study, we embarked on a modelling approach and developed different mathematical simulators using the Comsol multi-physics mathematical package, which is based on finite element methods. The finite element technique has been proved to be a suitable choice for the complex flow geometry, and is widely applied by many authors ((Furui et al., 2003), ( Jamiolahmady et al., 2005), (Karakas and Tariq, 1991); (Klotz et al., 1974)). It should be noted that simulating such three-dimensional well geometry using a commercial compositional simulator for the large number of simulations required to cover the required wide range of variation of pertinent parameters proved to be quite cumbersome. We also experienced a lot of convergence problems due to numerical instability when we initially considered a commercial reservoir simulator. The adaptive mesh generator of the Comsol mathematical package was tuned to generate a fine mesh that secures the accuracy and precision of the results.

\section{Three-Dimensional Horizontal Well In-House Simulators}

The three-dimensional system considered in this study consists of a horizontal well with wellbore radius $\left(\mathrm{r}_{\mathrm{w}}\right)$ and length (L), in a single layer cubic reservoir, as shown in Figure 1. This homogenous porous medium has an absolute permeability (k) and a formation thickness (h). The length of the reservoir in the $\mathrm{x}$ and $\mathrm{y}$ directions is assumed to be 2.5 times the length of the horizontal well. It has been shown (Ghahri P., 2010) that the impact of partial penetration on the geometric skin and the productivity of horizontal wells is negligible for Xres/L $>1.5$.

The equations employed in this study are similar to those described for gas condensate flow in a perforated region (Jamiolahmady et al., 2007) as presented in Appendix B. The methodology and boundary conditions are also described in Appendix B. 
Due to the existing symmetry, only a quarter of the model has been considered in this study. This reduces the computation time and facilitates having a high quality mesh. Mesh quality is the most important parameter of mesh design, which affects both the accuracy and convergence of a numerical solution. Considerable attempts

113 were made to generate consistent high quality mesh files for different 3D horizontal well geometries. We have

114 secured the accuracy of the results of our horizontal well models by selecting a high quality mesh as well as quadratic interpolation shape functions, which approximate the partial differential equation.

\section{Reservoir Fluid}

The composition and fluid properties of equilibrated phases of a fixed overall composition depend only on the pressure for a given temperature. In this study, binary moderate ( 0.74 of $\mathrm{C} 1$ (methane) \& 0.26 of n-C4 (normal butane)) and rich ( 0.88 of $\mathrm{C} 1$ and 0.12 of $\mathrm{C} 10$ (decane)) gas condensate fluid models were used to validate the integrity of the proposed approach. The maximum liquid drop out of the Constant Volume Depletion (CVD) test of these moderate and rich gas condensate fluid models are $23 \%$ and $30 \%$, respectively. The values of composition, density $(\rho)$, viscosity $(\mu)$ and interfacial tension (IFT) of the $\mathrm{C} 1-\mathrm{nC} 4$ mixtures which were implemented in the model are those measured in the gas condensate group laboratory as well as literature data ((Sage et al., 1940); SUPERTRAPP (Ely and Huber, 1992); (Weinaug and Katz, 1943)) at $311 \mathrm{~K}$ over a wide pressure range. For the selected C1-C10 rich gas condensate fluid model, we used the PVTi option of the ECLIPSE simulator to generate the PVT data.

\section{Validation Of The Horizontal Well In-House Simulator}

130 The accuracy of the two phase mathematical in-house simulator (Comsol) was confirmed by comparing some of its results with those of ECLIPSE300 (GeoQuest) at the same prevailing flow conditions.

The reservoir model in this exercise had the rock properties of Texas Cream with porosity of 0.21 and permeability of $9.1 \mathrm{mD}$. The reservoir fluid was a binary mixture of $\mathrm{C} 1$ (methane) and $\mathrm{n}-\mathrm{C} 4$ (normal butane) as described in the previous section. The reservoir dimensions were $38 \mathrm{~m}$ in the $\mathrm{x}$ and $\mathrm{y}$ directions and $4 \mathrm{~m}$ in the $\mathrm{z}$ direction. The horizontal well length was $15 \mathrm{~m}$. The ECLIPSE and Comsol reservoir models are presented in

136 Figure 2. Many different cases were simulated using both the ECLIPSE300 (GeoQuest) and the in-house simulators. The fractional flow at average reservoir pressure was the same in both simulators. A very fine grid was used to capture the abrupt changes in the flow parameters near the wellbore. As the $\mathrm{k}_{\mathrm{r}}$ correlations used in these two models (i.e. Comsol \& ECLIPSE300) are different, the base curve relative permeability was used to describe flow around the horizontal well. This is the relative permeability curve measured at a high IFT (above which $\mathrm{kr}$ is independent of coupling) and low velocity (below which $\mathrm{kr}$ is independent of coupling and inertial effects).

In the ECLIPSE 300 model, seventy injection wells were placed at the boundary of the reservoir to keep the reservoir pressure constant at the drainage boundary.

Figure 3 shows the good agreement between the two results. The arithmetic average absolute percentage deviation (AAD\%) of the flow rates predicted by the ECLIPSE300 simulator (GeoQuest) compared to those of the horizontal well simulator was $2.9 \%$. 
3. A NEW MODEL (AN IDEAL OPEN HOLE) FOR HORIZONTAL WELL PRODUCTIVITY CALCULATION

A horizontal well usually experiences less pressure drop compared to a vertical open hole system. This pressure change is usually quantified in terms of a skin, or an effective wellbore radius, included in the well productivity calculation of an equivalent open hole system. The combined effects of all the skin factors due to geometry, formation damage, perforation and high velocity (non-Darcy), etc. is referred to as a total skin, $\mathrm{S}_{\mathrm{t}}$.

Therefore a horizontal well productivity is expressed by

$$
Q=\frac{2 \pi k h \Delta p}{\operatorname{Ln}\left(\frac{r_{e}}{r_{w}}\right)+S_{T}}=\frac{2 \pi k h \Delta p}{\operatorname{Ln}\left(\frac{r_{e}}{r_{w}^{\prime}}\right)} .
$$

Here, the drainage volumes of the horizontal and its equivalent open hole system are assumed to be equal $\left(\mathrm{V}_{\mathrm{h}}=\right.$ $\left.158 \quad \mathrm{~V}_{\mathrm{v}}\right)$.

The effective wellbore radius of an open hole is defined here as follows:

$$
r_{w}^{\prime}=r_{w} \times e^{-S_{T}} .
$$

where $S_{t}$ is the total skin. For single phase Darcy flow without formation damage, the total skin is equal to the geometric skin, $\mathrm{S}_{\mathrm{t}=\mathrm{S}_{\mathrm{g}}}$.

In an earlier study, the author developed a correlation for geometric skin $\left(S_{g}\right)$, which correctly accounts for the impact of horizontal well geometry on the flow, using the efficient statistical method response surface method (P. Ghahri et al. 2009). The integrity and accurately of the proposed formulation was validated against a developed mathematical numerical model and other formulations available in the literature, which have limited range of application. Accordingly, the formula is very reliable for a wide range of variation of pertinent parameters.

However, it should be noted that the above formulation is only applicable for single phase flow horizontal well productivity and is not capable of explicitly predicting the productivity of two phase flow of gas and condensate or single phase non-Darcy flow. The computation of the effective wellbore radius is not straightforward because of the difference in the impact of the phase change and the velocity and IFT effects on the performance of the vertical and horizontal two flow systems. Therefore, the effective wellbore radius for such cases, should not only be a function of wellbore geometry, but also an implicit function of volumetric flow rate and fluid properties.

In this study, the main objective is to propose a formulation for the effective wellbore radius, which accounts for the combined effects of geometrical parameters (i.e. wellbore radius, reservoir thickness and horizontal well length) as well as flow parameters (i.e. IFT, velocity and fluid properties) on the horizontal well productivity.

The proposed semi analytical model predicts the horizontal well productivity of single phase non-Darcy flow and two phase flow of gas and condensate based on an equivalent open hole model with an effective wellbore radius.

181 The pseudo pressure concept is used to extend the single phase horizontal well productivity calculation to the two phase flow of gas and condensate. 


$$
m=\frac{2 \pi k h \Delta m(p)}{\operatorname{Ln}\left(\frac{r_{e}}{r_{w}}\right)+S_{T}}=\frac{2 \pi k h \Delta m(p)}{\operatorname{Ln}\left(\frac{r_{e}}{r_{w}^{\prime}}\right)},
$$

where $\mathrm{m}(\mathrm{p})$ is the pseudo pressure function defined for two phase flow of gas and condensate as:

$$
m(p)=\int_{p_{w}}^{p_{e}}\left(\frac{\rho_{g} k_{r g}}{\mu_{g}}+\frac{\rho_{c} k_{r c}}{\mu_{c}}\right) d p .
$$

Here, $\mathrm{m}$ is the mass flow rate, $\mathrm{p}_{\mathrm{w}}$ and $\mathrm{p}_{\mathrm{e}}$ are the wellbore and external pressures. $\rho, \mu$ and $k_{r}$ are the density, viscosity and relative permeability. The subscripts $g$ and $\mathrm{c}$ refer to gas and condensate.

As will be explained later, a formulation for the effective wellbore radius has been proposed here which accounts for the combined effects of the well geometry, IFT, velocity and fluid properties on the horizontally well productivity.

The details of solving Equation 3 for an open hole using finite difference method and pseudo pressure has been described in Appendix C. This solution can be easily done using an excel spread sheet for different number of grids.

\section{Effective Wellbore Radius Formulation}

In a systemic approach, a model is first proposed, which simulates the steady state single phase non-Darcy flow, including the inertial effect on the well productivity calculation. Then the proposed model is extended for a two phase of gas and condensate.

\section{Single Phase Non-Darcy Flow}

In the in-house simulator the two phase $\mathrm{kr}(\mathrm{krg}$ and $\mathrm{krc})$ formulation is converted to single phase non Darcy by substituting GTR=1 in the equation A-7, as explained in Appendix A. The two phase pseudo pressure function in Equation 3 is also converted to the single phase pseudo pressure function as follow:

$$
m(p)=\int_{p_{w}}^{p_{e}}\left(\frac{\rho_{g} k_{r g}}{\mu_{g}}\right) d p
$$

$$
k_{r g}=\frac{1}{1+\mathrm{Re}}
$$

$$
\operatorname{Re}=\frac{\rho|V| k \beta}{\mu} \text {. }
$$

$\rho, \mathrm{V}, \mathrm{k}, \beta$ and $\mu$ refer to the single-phase density, velocity, permeability, inertia factor and viscosity. 
It should be noted that applying Equations 6 and 5 in Darcy Law will correctly convert the Darcy equation to the Forchhiemer equation, which describes single phase non-Darcy inertial flow. In other words, the inertial effect is defined as a single phase relative permeability (Equation 5). As the velocity decreases, for very long horizontal wells for instance, the single phase relative permeability $\left(\mathrm{k}_{\mathrm{rg}}\right)$ approaches to 1 , reducing the equation to the Darcy flow equation. However, for predicting the productivity of short to medium length horizontal wells, where the inertia reduces the productivity significantly, $\mathrm{k}_{\mathrm{r}}$ would be less than one and the effective well bore radius should be corrected to account for the high velocity inertial effect (P. Ghahri 2010).

To obtain an appropriate formulation for $r_{w}$, the mass flow rates of the 1D equivalent open hole model, EOH,

(Equation 3) and those of 3D horizontal well in-house simulators were matched iteratively by varying $r_{w}^{\prime}$. and 8) and the single phase inertial factor express the impact of all pertinent parameters.

$$
L_{D}=\frac{L}{h}
$$

$h_{D}=\frac{h}{r_{w}}$

$\mathrm{L}, \mathrm{h}$, and $\mathrm{r}_{\mathrm{w}}$ are the horizontal well length, reservoir thickness and wellbore radius.

Further analysis showed that the effective wellbore radius can be represented by Equation 9 .

$$
r_{w}^{\prime}=\frac{r_{w} \times e^{-S g}}{1+a \times\left(\frac{\operatorname{Re} \times h_{D}}{L_{D}}\right)^{b}}
$$

$\mathrm{S}_{\mathrm{g}}$ is the geometric skin and can easily be calculated assuming single phase gas Darcy flow around the horizontal well using any available formulation in the literature (i.e. Joshi, Borosiv, Economides, Goode, P. and Kuchuk, Babu, D. and Odeh, Ghahri et. al (2009), etc ). The above equation is very simple and easy to use, i.e. there are only two coefficients ( $a$ and $b$ ) that need to be estimated. Here, two sets of data were generated based on the basic physical properties of two different rocks used to confirm the integrity of the proposed formulation. The effective wellbore radius varied substantially when the reservoir permeability was varied from 11 to $110 \mathrm{mD}$, for the same pressure drop, as the velocity was significantly higher for the latter. The coefficients a and $b$ were estimated as 0.04 and 0.6 respectively, using one of these sets of data; i.e. the set of data referred to as HW-1 in Table 1. The other data set, referred to as HW-2 in Table 1, was used to verify the reliability of the correlation as described below.

Figure 4 confirms the accuracy of the developed flow skin equation by comparing the calculated mass flow rate obtained using an equivalent open hole, in which the developed $r_{w}^{\prime}$ formulation, Equation 9, and the iterative procedure described in Appendix $\mathrm{C}$ have been incorporated, with those of the horizontal well in-house simulator. The reservoir models and the range of velocities are those listed in Table 1, the HW-1 data set. The AAD\% (average absolute deviation error) for 270 data points in this study is only $2 \%$.

The accuracy of the developed effective wellbore radius formulation was further verified by applying the proposed method to another a reservoir model, with different permeability and velocity with AAD $\%$ of $2 \%$. Here, 
of horizontal well length, wellbore radius and thickness of the reservoir were covered for the 220 data points as shown in the HW-2 data set (Table 1).

\section{Two Phase Flow Of Gas and Condensate}

In this part, we followed an approach similar to that mentioned above and proposed a general formulation for the calculation of the effective wellbore radius of an equivalent open hole system for two phase flow of gas condensate, which correctly extends to that of single phase when the gas fractional flow is unity $(G T R=1)$ and which produces the same flow performance, as that of a 3D horizontal well system.

To obtain an appropriate formulation for $r_{w}^{\prime}$, the mass flow rates of the two phase open hole model (Equation 3) and that of 3D two phase horizontal well in-house simulators were matched iteratively by varying $r_{w}^{\prime}$.

This was done for a large bank of data, containing over 1300 data points. For this part of study, the Texas Cream and Berea described the reservoir rock properties. The two fluids were binary mixtures of $\mathrm{C} 1$ and $\mathrm{C} 4$ and $\mathrm{C} 1$ and $\mathrm{C} 10$ introduced above. The variation of the reservoir pressure, wellbore pressure and GTR (Ttal gas fractional flow) are given in Table 2. The other characteristic of different models used here are also listed in Table 2.

For a gas condensate system, the flow regime is controlled by coupling, operating at moderate to high velocities, and inertia, operating at high velocities. These two act in opposite directions, i.e. the former improves the flow performance whilst the latter reduces the flow efficiency.

Figures 5 and 6 show pressure and condensate saturation distribution maps in a drainage area of a horizontal well. It should be noted that the employed generalized $k_{r}$ correlation (Jamiolahmady et al. 2009) generates $k_{r}$ at different velocity and IFT, with the relative permeability ratio $\left(\mathrm{k}_{\mathrm{rgrt}}=\mathrm{k}_{\mathrm{rg}} /\left(\mathrm{k}_{\mathrm{rg}}+\mathrm{k}_{\mathrm{rc}}\right)\right)$ rather than local saturation as the main independent. However, the values of saturation as a function of $\mathrm{k}_{\mathrm{rgtr}}$ are known for the base $\mathrm{k}_{\mathrm{r}}$ curve. Considering that saturation values do not enter into the pseudo-pressure calculation, these saturation values are assigned to the corresponding $\mathrm{k}_{\mathrm{rgtr}}$ at any time and location: that is, the saturation map across the reservoir is calculated by knowing $\mathrm{k}_{\mathrm{rgtr}}$ across the reservoir. It is clear that the shapes of condensate and pressure profile distributions are similar.

For two phase flow of gas and condensate, the important parameters, which affect well productivity, are density, viscosity and relative permeability of both flowing (gas and condensate) phases. Mass mobility, expressed by Equation 10, is used to express the effect of these three important parameters on the effective wellbore radius.

$$
\bar{M}_{r}=\frac{\rho_{g} k_{r g}}{\mu_{g}}+\frac{\rho_{c} k_{r c}}{\mu_{c}},
$$

where $\frac{\rho_{g} k_{r g}}{\mu_{g}}$ and $\frac{\rho_{c} k_{r c}}{\mu_{c}}$ are the absolute mass mobility for gas and condensate, respectively.

We intend to use an average value of mass mobility to modify and extend Equation 9 to gas condensate systems. Here we multiply Equation 9 by the ratio of (two phase gas and condensate) mass mobility of the actual flow to that of the base case. For this latter mass mobility term, the base relative permeability is used. Hence, Equation 5 can be rewritten as: 


$$
r_{w}^{\prime}=\frac{r_{w} \times e^{-S m}}{1+a \times \frac{\operatorname{Re} \times h_{D}}{L_{D}}} \times\left(\frac{\bar{M}_{r E O H}}{\left.\bar{M}_{r H W}\right)_{D a r c y}}\right)_{\text {ave }}=\frac{r_{w} \times e^{-S m}}{1+a \times \frac{\operatorname{Re} \times h_{D}}{L_{D}}} \times\left(\frac{\bar{M}_{r E O H}}{\left.\bar{M}_{r E O H}\right)_{\text {Darcy }}}\right)_{\text {ave }}
$$

280

281

282

283

284

285

286

287

288

289

290

291

where $\bar{M}_{r E O H}$ and $\left.\bar{M}_{r E O H}\right)_{D a r c y}$ are the average mass mobility for EOH with and without considering the coupling and inertial effects, respectively. Or in another form, Equation 11 can be rewritten as:

$$
r_{w}^{\prime}=\frac{r_{w} \times e^{-S m}}{1+a \times\left(\frac{\operatorname{Re} \times h_{D}}{L_{D}}\right)^{b}} \times\left(\frac{\left(\frac{\rho_{g} k_{r g}}{\mu_{g}}+\frac{\rho_{c} k_{r c}}{\mu_{c}}\right)_{E O H}}{\left(\frac{\rho_{g} k_{r g}}{\mu_{g}}+\frac{\rho_{c} k_{r c}}{\mu_{c}}\right)_{E O H-D a r c y}}\right)_{a v e}=\frac{r_{w} \times e^{-S m}}{1+a \times\left(\frac{\operatorname{Re} \times h_{D}}{L_{D}}\right)^{b}}
$$

$$
\times\left(\frac{\left(\frac{\rho_{g} k_{r g}}{\mu_{g}}+\frac{\rho_{c} k_{r c}}{\mu_{c}}\right)_{E O H}}{\left(\frac{\rho_{g} k_{r g b}}{\mu_{g}}+\frac{\rho_{c} k_{r c b}}{\mu_{c}}\right)_{E O H}}\right)_{a v e}
$$

The average mobility ratio term depends on the fluid properties and relative permeability across the reservoir drainage area. It is important to point out that the above formulation includes the gas and condensate relative permeability and hence the impact of velocity and IFT in the form of coupling and inertial effects has been included. We have noted that this formula expresses the horizontal well performance accurately, but it is possible to make it simpler for practical purposes.

Based on a pseudo-pressure calculation approach, the condensate relative permeability can be estimated by the definition of fractional flow using the following equation (Jamiolahmady et. al. 2006):

$$
k_{r c}=k_{r g} \times \frac{1-G T R}{G T R} \times \frac{\mu_{c}}{\mu_{g}},
$$

where GTR, $\mu_{c}$ and $\mu_{g}$ are the total gas fraction, condensate and gas viscosity, respectively. Substituting the above equation into Equation 11 results in:

$$
r_{w}^{\prime}=\frac{r_{w} \times e^{-S m}}{1+a \times\left(\frac{\operatorname{Re} \times h_{D}}{L_{D}}\right)^{b}} \times\left(\frac{\left(\frac{k_{r g}}{\mu_{g}}\left(\rho_{g}+\rho_{c} \times \frac{1-G T R}{G T R}\right)_{E O H}\right)_{a v e}}{\left(\frac{k_{r g b}}{\mu_{g}}\left(\rho_{g}+\rho_{c} \times \frac{1-G T R}{G T R}\right)_{E O H}\right)_{a v e}}\right),
$$

Rearranging Equation 14 gives:

$$
r_{w}^{\prime}=\frac{r_{w} \times e^{-S m}}{1+a \times\left(\frac{\operatorname{Re} \times h_{D}}{L_{D}}\right)^{b}} \times\left(\frac{\left(\frac{k_{r g}}{\mu_{g} \times G T R}\left(\rho_{g} \times G T R+\rho_{c} \times(1-G T R)\right)_{E O H}\right)_{a v e}}{\left(\frac{k_{r g b}}{\mu_{g} \times G T R}\left(\rho_{g} \times G T R+\rho_{c} \times(1-G T R)\right)_{E O H}\right)_{\text {ave }}}\right),
$$

or 


$$
r_{w}^{\prime}=\frac{r_{w} \times e^{-S m}}{1+a \times\left(\frac{\operatorname{Re} \times h_{D}}{L_{D}}\right)^{b}} \times\left(\frac{\left(\frac{k_{r g}}{\mu_{g} \times G T R}\left(\bar{\rho}_{a v e}\right)_{E O H}\right)_{a v e}}{\left(\frac{k_{r g b}}{\mu_{g} \times G T R}\left(\bar{\rho}_{\text {ave }}\right)_{E O H}\right)_{a v e}}\right),
$$
$\frac{\left(k_{r g}\right)_{\text {EOHave }}}{\left(k_{r g b}\right)_{\text {EOHave }}}$ could be approximately predicted by an average value corresponding to that around the wellbore.

where $\bar{\rho}_{a v e}$ is the volumetric average density of two phase gas and condensate which is defined by:

$$
\bar{\rho}_{\text {ave }}=\rho_{g} \times G T R+\rho_{c} \times(1-G T R),
$$

The fluid properties and GTR variation in the reservoir depend on the pressure profile for a fluid with fixed total composition. We assume that the pressures and GTR values are the same at the wellbore and exterior boundaries for both the base case and that affected by coupling and inertia. Furthermore, it has previously been shown that the effect of fluid properties on the performance of a perforated well compared to that of an equivalent open hole well, both flowing under same pressure drop and with the same GTR, is minimal (Jamiolahmady et al. 2005). This simplifying assumption implies that the mobility ratio can approximately be estimated by the following equation

$$
\left(\frac{\left(\frac{\mathrm{k}_{\mathrm{rg}}}{\mu_{\mathrm{g}} \times \mathrm{GTR}}\left(\bar{\rho}_{\mathrm{ave}}\right)_{\mathrm{EOH}}\right)_{\mathrm{ave}}}{\left(\frac{\mathrm{k}_{\mathrm{rgb}}}{\mu_{\mathrm{g}} \times \mathrm{GTR}}\left(\bar{\rho}_{\mathrm{ave}}\right)_{\mathrm{EOH}}\right)_{\mathrm{ave}}}\right) \cong \frac{\left(\mathrm{k}_{\mathrm{rg}}\right)_{\mathrm{EOH}_{\mathrm{ave}}}}{\left(\mathrm{k}_{\mathrm{rgb}}\right)_{\mathrm{EOH}_{\mathrm{ave}}}},
$$

Figures 5 and 6, show the base and affected (by coupling and inertia) gas relative permeability for an equivalent open hole systems for horizontal well-3 and horizontal well-4, defined in Table 2. It is noted that gas relative permeability $\left(\mathrm{k}_{\mathrm{rg}}\right)$ values change mostly around the wellbore, where coupling and inertia are important. Far away from the wellbore, the difference between these two curves, $\mathrm{k}_{\mathrm{rgb}}$ and $\mathrm{k}_{\mathrm{rg}}$, is almost constant. Thus Therefore the following equation can be used to estimate the effective wellbore radius:

$$
r_{w}^{\prime}=\frac{r_{w} \times e^{-S m}}{1+a \times\left(\frac{\operatorname{Re} \times h_{D}}{L_{D}}\right)^{b}} \times \frac{k_{r g}}{k_{r g b}} .
$$

It should be noted that Equation 19 can be applied for both single phase and two phase, gas and condensate, flow cases. That is, for the single phase flow case, $\mathrm{k}_{\mathrm{r}}=1.0$ and Equation 19 converts to Equation 9. As noted earlier, the above proposed formulations for the effective wellbore radius (Equations 19) and pseudo pressure function (Equations 3 and 4) are both functions of fluid properties and flow rates. Consequently, the productivity calculation requires an iterative procedure. The details of this procedure have been explained in Appendix C.

Figures 7 and 8 confirm the accuracy of the proposed approach by comparing the calculated mass flow rates obtained using an equivalent open hole radius based on the proposed approach with those of the 3D horizontal 
well in-house simulator. In this exercise wide ranges of the GTR, pressure and horizontal well geometric variables values were considered, as listed in Table 2.

\section{APPLICATION OF NEW MODEL FOR THE HORIZONTAL WELL PRODUCTIVITY PRODUCTION}

The added value of the proposed approach has been demonstrated by studying the impact of pertinent parameters; well length, reservoir thickness and gas fractional flow on horizontal well productivity using an excel spreadsheet (1D simulator) which implements the new model formulation (as explained in Appendix D). For all cases presented here, the fluid was $\mathrm{C} 1-\mathrm{nC} 4$ and the reservoir rock properties were those of $\mathrm{HW}-1$ are cases as listed in Table 2. The reservoir drainage and wellbore radius are 200 and $0.1 \mathrm{~m}$, respectively. The differential pressure across the reservoir model is $300 \mathrm{psi}$, unless otherwise stated.

It should be noted that, performing the simulation corresponding to ach point shown in Figures 9 to 11, using 3-D compositional simulator as an alternative tool will be quite time consuming and cumbersome, especially if there are convergence problems.

\section{Well Length}

For these simulations, the pressure at the wellbore and the differential pressure across the reservoir model were 1500 and 300 psi, respectively. For all the cases presented here, the well productivity is calculated both with and without coupling and inertia (referred to as NI and NCI, respectively). It should be noted that, for the well productivity without coupling and inertia, the base curve relative permeability is used. As can be seen from Figure 9 , the well productivity increases when the effect of inertia and coupling are taken into consideration. Furthermore, coupling has a more pronounced effect on well productivity for a shorter horizontal well.

\section{Reservoir Thickness}

The effect of reservoir thickness on the productivity of a horizontal well with CI effects is shown in Figure 10. The reservoir, well dimensions, rock and fluid properties used here are those for Figure 9.

It is noted that the well productivity is greater for the thicker reservoir model; that is, the drainage volume for flow of a thick reservoir model compared to that in a thin reservoir is much greater. Furthermore, as the horizontal length (L) increases, the difference between the mass flow rates for thick and thin reservoirs increases substantially.

\section{Gas Fractional Flow}

Figure 11 shows the effect of gas to total flow rate ratio $\left(\mathrm{GTR}_{\mathrm{w}}\right)$ on the productivity of a horizontal well, with CI effects. For the set of the productivity calculations presented in Figure 11, the horizontal well length is $50 \mathrm{~m}$. As can be seen, increasing $\mathrm{GTR}_{\mathrm{w}}$ decreases the well productivity, due to the more pronounced effect of inertia.

\section{SUMMARY AND CONCLUSIONS}


A number of in-house simulators were developed to simulate the performance of horizontal wells in gas and gas condensate reservoirs for steady state conditions. Using the results of these simulators, a generalized effective wellbore radius formulation for an equivalent open hole system was developed which replicate the flow performance of the corresponding complete 3-D system accounts for coupling, inertia and compositional effects. The accuracy of this approach was confirmed by comparing the results with corresponding values obtained using the 3-D horizontal well in-house simulator.

Due to the presence of velocity in this formulation, its implementation requires an iterative procedure. However, the small number of iterations required confirms the validity of the assumptions and the reliability of the proposed approach.

The ease and added value of the application of the proposed approach has been demonstrated by studying the impact of well length, reservoir thickness and gas fractional flow using an excel spreadsheet that performs implement this calculation. The results, which are similar to those of the 3D simulator, albeit with much less calculation, demonstrate that: (i) coupling has a more pronounced effect on well productivity for the horizontal well with shorter length, (ii) well productivity is greater for the thicker reservoir, especially for the longer horizontal well, and (iii) increasing gas fractional flow decreases the well productivity, due to the more pronounced effect of inertia.

These results confirm that the proposed method can be used to estimate horizontal well productivity and optimize well characteristics for the two phase flow of gas and condensate using a simple excel spreadsheet, which makes it attractive for practical purposes.

\section{REFERENCES}

Babu, D. and Odeh, A.S., 1989. Productivity of a horizontal well. SPE (Society of Petroleum Engineers) Reservoir Engineering;(USA), 4(4).

Babuska, I. and Suri, M., 1990. The p-and hp versions of the finite element method, an overview. Computer methods in applied mechanics and engineering, 80(1-3): 5-26.

Babuska, I., Szabo, B.A. and Katz, I.N., 1981. The p-version of the finite element method. SIAM journal on numerical analysis, 18(3): 515-545.

Borisov, J.P., 1964. Oil production using horizontal and multiple deviation wells. Nedra, Moscow.

Comsol, A., 2005. COMSOL multiphysics user's guide. Version: September.

Dehane, A. and Tiab, D., 2000. Performance of Horizontal Wells in Gas Condensate Reservoirs, Djebel Bissa Field, Algeria.

Economides, M., Brand, C. and Frick, T., 1996. Well Configurations in Anisotropic Reservoirs. SPEFE 11 (4): 257-262, SPE-27980-PA. DOI: 10.2118/27980-PA.

Elgaghah, S., Osisanya, S. and Tiab, D., 1996. A simple productivity equation for horizontal wells based on drainage area concept.

Ely, J. and Huber, M., 1992. NIST Thermophysical Properties of Hydrocarbon Mixtures Database (SUPERTRAPP) Version 1.0 Users' Guide. National Institute of Standards and Technology.

Escobar, F.H. and Montealegre, M., 2008. A More Accurate Correlation for the Productivity Index of Horizontal Wells. J. Eng. Applied Sci, 3(1): 70-78. 
Forchheimer, P., 1914a. Hydraulik. BG Teubner.

398 Forchheimer, P., 1914b. Hydraulik: Leipzig and Berlin. BG.

399 Furui, K., Zhu, D. and Hill, A., 2003. A Comprehensive Model of Horizontal Well Completion Performance.

400 GeoQuest, S., ECLIPSE Reference Manual. 2005A.

401 Ghahri, P., 2010. Modelling of Gas-condensate flow around horizontal and deviated wells and cleanup efficiency of hydraulically fractured wells.

403 Giger, F., Reiss, L. and Jourdan, A., 1984. The Reservoir Engineering Aspect of Horizontal Drilling," paper SPE 13024, pp. 16-19.

Goode, P. and Kuchuk, F., 1991. Inflow performance of horizontal wells. SPE Reservoir Engineering, 6(3): 319323.

Harris, M., 1966. The Effect of Perforating Oil Well Productivity. Journal of Petroleum Technology, 18(4): 518528.

Hashemi, A. and Gringarten, A., 2005. 94178-Comparison of Well Productivity Between Vertical, Horizontal and Hydraulically Fractured Wells in Gas-Condensate Reservoirs.

Helmy, M. and Wattenbarger, R., 1998. Simplified productivity equations for horizontal wells producing at constant rate and constant pressure.

413 Hughes, T.J.R., 1987. The finite element method. Prentice-Hall intern. Englewood Cliffs (NJ).

414 Jamiolahmady, M. and Danesh, A., 2007. Comparison of Vertical, Slanted, and Horizontal Wells Productivity in Layered Gas-Condensate Reservoirs.

416 Jamiolahmady, M., Danesh, A., Sohrabi, M. and Ataei, R., 2007. Gas-Condensate Flow in Perforated Regions. SPE Journal, 12(1): 89-99.

418 Jamiolahmady, M., Danesh, A., Sohrabi, M. and Duncan, D., 2006. Flow around a rock perforation surrounded by crushed zone: Experiments vs. theory. Journal of Petroleum Science and Engineering, 50(2): 102114.

Jamiolahmady, M., Danesh, A., Tehrani, D. and Duncan, D., 2000. A mechanistic model of gas-condensate flow in pores. Transport in porous media, 41(1): 17-46.

Jamiolahmady, M., Sohrabi, M., Ireland, S. and Ghahri, P., 2009. A generalized correlation for predicting gascondensate relative permeability at near wellbore conditions. Journal of Petroleum Science and Engineering, 66(3-4): 98-110.

426 Joshi, S.D., 1991. Horizontal well technology. Pennwell Corp.

427 Karakas, M. and Tariq, S., 1991. Semianalytical productivity models for perforated completions. SPE Production Engineering, 6(1): 73-82.

Klotz, J., Krueger, R. and Pye, D., 1974. Effect of perforation damage on well productivity. Journal of Petroleum Technology, 26(11): 1303-1314.

Locke, S., 1981. Advanced method for predicting the productivity ratio of a perforated well. J. Pet. Technol.;(United States), 33(12).

433 Lu, J., 2001. New productivity formulae of horizontal wells. Journal of Canadian Petroleum Technology, 40(10).

434 Lu, J. and Tiab, D., 2007. A Simple Productivity Equation for a Horizontal Well in Pseudosteady State in a Closed 
Marir, B. and Djebbar, T., 2006. Performance of Horizontal Wells In Gas Condensate Reservoirs: Hassi R'Mel, Algeria.

438

Myers, R. and Montgomery, D., Response surface methodology: process and product optimization using designed experiments, 1995. Wileys, NY.

440 Permadi, P., 1995. Practical methods to forecast production performance of horizontal wells.

441 Renard, G. and Dupuy, J., 1991. Formation Damage Effects on Horizontal-Well Flow Efficiency (includes associated papers 23526 and 23833 and 23839). Journal of Petroleum Technology, 43(7): 786-789.

443 Sage, R., Hicks, B. and Lacey, W., 1940. Phase equilibria in hydrocarbon systems. Industrial \& Engineering Chemistry, 32(8): 1085-1092.

445 Sheng and J, 2007. Discussion of Permeability Anisotropy Effect, SPE 114504.

446 Wang, S. and Eaton, J., 2007. Predicting Productivity Index of Horizontal Wells. Journal of Energy Resources

448 Weinaug, C.F. and Katz, D.L., 1943. Surface tensions of methane-propane mixtures. Industrial \& Engineering 
453

454

455

456

457

458

459

460

461

462

463

464

465

466

467

468

469

470

471

472

473

474

475

476

\section{Appendix A- Generalised Relative Permeability Expression}

In this section, a summary of the $\mathrm{k}_{\mathrm{r}}$ correlation used in the current work is presented. More detailed information on the structure of the correlation and derivation of formulations can be found elsewhere (Jamiolahmady et al, 2009).

The gas relative permeability, $\mathrm{k}_{\mathrm{rg}}$, is interpolated between the base curve and the miscible-fluids curve using an interpolation function $Y_{j}$ :

$\mathrm{k}_{\mathrm{rg}}=\mathrm{Y}_{\mathrm{g}}\left(\mathrm{k}_{\mathrm{rgb}}\right)_{\text {iner }}+\left(1-\mathrm{Y}_{\mathrm{g}}\right)\left(\mathrm{k}_{\mathrm{rgm}}\right)_{\text {iner }}$.

The miscible and the base curve in Eq. A-1 are based on the relative permeability ratio as the main variable. The relative permeability ratio is defined as

$\mathrm{k}_{\mathrm{rgtr}}=\frac{\mathrm{k}_{\mathrm{rg}}}{\mathrm{k}_{\mathrm{rg}}+\mathrm{k}_{\mathrm{rc}}}=\frac{1}{1+\left(\frac{\mathrm{k}_{\mathrm{rc}}}{\mathrm{k}_{\mathrm{rg}}}\right)}$

Solving Eq. A-2 for condensate relative permeability $\left(\mathrm{k}_{\mathrm{rc}}\right)$ gives

$\mathrm{k}_{\mathrm{rc}}=\frac{\mathrm{k}_{\mathrm{rg}}\left(1-\mathrm{k}_{\mathrm{rgtr}}\right)}{\mathrm{k}_{\mathrm{rgtr}}}$.

Therefore, when $\mathrm{k}_{\mathrm{rg}}$ is determined as a function of $\mathrm{k}_{\mathrm{rgtr}}$, this equation automatically gives the corresponding $\mathrm{k}_{\mathrm{rc}}$ at the same $\mathrm{k}_{\mathrm{rgtr}}$.

It is noted that the $\mathrm{k}_{\mathrm{rgtr}}$ ratio could be calculated in terms of gas fractional flow (GTR), using the extended form of the Darcy's law for two phase flow, without the need for further information.

$\mathrm{k}_{\mathrm{rgtr}}=\frac{\mathrm{k}_{\mathrm{rg}}}{\mathrm{k}_{\mathrm{rc}}+\mathrm{k}_{\mathrm{rg}}}=\frac{\mu_{\mathrm{g}} \mathrm{GTR}}{\mu_{\mathrm{g}} \mathrm{GTR}+\mu_{\mathrm{c}}(1-\mathrm{GTR})}$

The gas fractional flow, GTR, is the ratio of the gas to the total (gas plus condensate) volumetric flow rate, Eq.

A-5.

$\mathrm{GTR}=\frac{\mathrm{Q}_{\mathrm{g}}}{\mathrm{Q}_{\mathrm{g}}+\mathrm{Q}_{\mathrm{c}}}=\frac{\mathrm{V}_{\mathrm{g}}}{\mathrm{V}_{\mathrm{c}}+\mathrm{V}_{\mathrm{g}}}$

GTR can be expressed in terms of CGFR using Eq. A-6.

GTR $=\frac{1}{1+\text { CGFR }}$

The base curve relative permeability curve is adjusted for the effect of inertia using the following equation:

$\left(k_{r g b}\right)_{\text {iner }}=\left(\frac{\left(k_{r g b}\right)_{\text {meas }}}{1+\frac{\beta\left[\rho_{\text {avg }}\right]_{b} k\left(k_{r g b}\right)_{\text {meas }}}{G T R \mu_{g b}}|V|_{T}}\right)$

where $\rho_{\text {ave }}$ is the average density based on the fractional flow of the two flowing phases. This equation has been obtained based on the momentum transfer rate for an equivalent phase, i.e. inertial pressure drop was 
calculated using the single phase inertial factor, total velocity and total (gas plus condensate) momentum inflow (Jamiolahmady et al. 2006 and 2009).

In Eq. A-7, $\left(\mathrm{k}_{\mathrm{rgb}}\right)_{\text {meas }}$ is the measured gas relative-permeability at specified base IFT of $>=3 \mathrm{mNm}^{-1}$ (below which $k_{r}$ is a function of IFT) and capillary number of $<=1 \mathrm{E}-7$ (above which $k_{r}$ is a function of velocity). Hereafter when we refer to base conditions we mean $\sigma_{b}=3$ and Ncb=1E-7. It is understood that if IFT $>3$ and $\mathrm{Ncb}<1 \mathrm{E}-7$ the base relative permeability would be the same. The base velocity in the laboratory test for measuring $\mathrm{k}_{\mathrm{rgb}}$ should be selected such that:

$$
\mathrm{Ncb}=\frac{\mu_{\mathrm{gb}}\left(\mathrm{V}_{\mathrm{gb}}+\mathrm{V}_{\mathrm{cb}}\right)}{\sigma_{\mathrm{b}}}
$$

The miscible gas relative permeability curve, which is modified to include the inertial effect, is calculated as follows:

$$
\left(\mathrm{k}_{\mathrm{rgm}}\right)_{\text {iner }}=\left(\frac{\mathrm{k}_{\mathrm{rgtr}}}{1+\left.\beta \rho_{\mathrm{m}}\left(\frac{\mathrm{k}}{\mu_{\mathrm{m}}}\right) \mathrm{V}\right|_{\mathrm{T}}}\right) .
$$

Similarly to Eq. A-7, this equation has been obtained based on the momentum transfer rate. If Eq. A-9 is added to the corresponding one for the condensate, one would obtain a $\mathrm{k}_{\mathrm{r}}$ term, which if included in Darcy Law gives the Forchheimer equation, Eq. 2, for the single phase flow conditions.

In Eq. A-9, the required miscible fluid properties (i.e. density, $\rho_{\mathrm{m}}$, and viscosity, $\mu_{\mathrm{m}}$ ) are the arithmetic average between the fluid properties of gas and liquid at any given pressure, in the vicinity of the miscible pressure.

It should be noted that, because of the presence of the rock properties ( $k$ and $\beta$ ) in Eqs. A-7 and A-9, there are different base and miscible relative permeability vs. $\mathrm{k}_{\mathrm{rgtr}}$ curves for each core at any velocity value.

Fig. A-1 shows the conventional base (corresponding to IFT=3 $\mathrm{mNm}^{-1}$ and $\mathrm{Ncb}=1 \mathrm{E}-7$, Eq. A-8) and miscible $\mathrm{k}_{\mathrm{rg}}$ ( $45^{\circ}$ degree diagonal line) and the corresponding $\left(\mathrm{k}_{\mathrm{rgb}}\right)_{\text {iner }}$ and $\left(\mathrm{k}_{\mathrm{rgm}}\right)_{\text {iner }}$ values affected by inertia, which have been calculated by Eqs. A-7 and A-9, respectively, at a velocity of $82.6 \mathrm{md}^{-1}$ for the RC3 core. It should be noted that because of the presence of the rock properties $(k$ and $\beta)$ in Eqs. A-7 and A-9, different $\left(k_{\mathrm{rgb}}\right)_{\text {iner }}$ and $\left(\mathrm{k}_{\mathrm{rgm}}\right)_{\text {iner }}$ vs. $\mathrm{k}_{\mathrm{rgtr}}$ curves at any velocity value, will be obtained for a different porous medium.

It should also be noted that although Eqs. A-7 and A-9 are for $S_{w i}=0$, the same relations are valid for $S_{w i}>0$, with $k_{\mathrm{eg}}\left(\mathrm{S}_{\mathrm{wi}}\right)$ and $\beta_{\mathrm{g}}\left(\mathrm{S}_{\mathrm{wi}}\right)$ replacing $\mathrm{k}$ and $\beta$, respectively.

The interpolation parameter, $\left(\mathrm{Y}_{\mathrm{g}}\right)$, mainly depends on the rock properties (i.e. $\left.\mathrm{k}, \phi, \beta\right)$, interfacial tension (IFT), pressure gradient, GTR, and the base capillary number as described in the original reference (Jamiolahmady et al. 2009).

The interpolation parameter $\left(\mathrm{Y}_{\mathrm{g}}\right)$ is expressed by Eq. A-10.

$$
Y_{g}=\frac{1+A_{1} x}{1+A_{1} x+A_{2} x^{2}}
$$

with 


$$
\mathrm{x}=\log \left[\sigma_{\mathrm{r}} \mathrm{Ncr}^{\prime}\right] \quad \mathrm{A}_{1}=-\frac{\mathrm{C}_{1}+\mathrm{C}_{3} \mathrm{~A}_{3}{ }^{\mathrm{C}_{4}}}{\mathrm{C}_{2}+\mathrm{A}_{3}{ }^{\mathrm{C}_{4}}} \quad \mathrm{~A}_{2}=\frac{\mathrm{C}_{1}+\mathrm{C}_{5} \mathrm{~A}_{3}{ }^{\mathrm{C}_{6}}}{\mathrm{C}_{2}+\mathrm{A}_{3}{ }^{\mathrm{C}_{6}}} \quad \mathrm{~A}_{3}=\beta \sqrt{\mathrm{k}},
$$

$$
\mathrm{C}_{1}=8, \mathrm{C}_{2}=800, \mathrm{C}_{3}=0.20, \mathrm{C}_{4}=2.20, \mathrm{C}_{5}=0.15, \mathrm{C}_{6}=0.81
$$

512 of base IFT $(\sigma)$ to current IFT value and $\mathrm{Ncr}^{\prime}$ refers to the ratio of $\mathrm{Nc}^{\prime}$, Eq. 5, at any condition to the 513 corresponding value for the base curve at the same $\mathrm{k}_{\text {rgtr. }}$. The base value of $\mathrm{Nc}^{\prime}$ at any $\mathrm{k}_{\mathrm{rgtr}}$ value can be obtained 514 by Eq. A-11, which requires the measured $\mathrm{k}_{\mathrm{rgb}}$, fluid properties and the corresponding base Nc of $1 \mathrm{E}-7$ defined 515 earlier (Eq. A-8),

$$
N c b^{\prime}=\frac{G T R}{\phi\left(k_{r g b}\right)_{\text {meas }}} N c b=\frac{G T R}{\phi\left(k_{r g b}\right)_{\text {meas }}} 1 E-7
$$

517 It is noted that $Y_{\mathrm{g}}$ mainly depends on the rock properties (i.e. $\mathrm{k}, \phi, \beta$ ), interfacial tension (IFT), pressure gradient,

518 GTR, and the base capillary number. It should be mentioned that in the $Y_{g}$ expression there is another term, which 519 accounts for the effect of micro-pores. The mercury porosimetry Pc curve of this core indicated that the contribution of micropores was minimal. Therefore, the corresponding additional term to express micro-pore effect has not been included here in Eq. A-10.

It is noted that Jamiolahmady et. al. (2006) had reported a $\mathrm{k}_{\mathrm{r}}$ correlation for gas condensate systems, which was based on fractional flow rate and expressed the combined effect of coupling and inertia. However, the dependency of relative permeability to fluid viscosity and lack of a proper definition of the lower limit of the correlation limited its use to the range of IFT values studied. That is, the reported base IFT of $0.85 \mathrm{mNm}^{-1}$ (above which $\mathrm{k}_{\mathrm{r}}$ is not a function of IFT), was not a universal value and the threshold capillary number fixing the lower limit of velocity (below which $\mathrm{k}_{\mathrm{r}}$ is not a function of velocity) was not identified. Furthermore, the inclusion of viscosity in the definition of independent variable made it difficult to fix the base conditions. In other words, the base $\mathrm{k}_{\mathrm{r}}$ curve reported as a function of fractional flow rate would be fluid dependent, i.e. it would vary with the pressure at which the measurements are conducted. This would contradict the definition of the base $\mathrm{k}_{\mathrm{r}}$ curve, which should be independent of pressure and velocity at which the measurement is conducted, as described above.

\section{Appendix B- Governing Flow Equations}

The equations employed in this study are similar to those described for gas condensate flow in a perforated region (Jamiolahmady et al., 2007).

536 For a single phase compressible fluid under steady state conditions the governing equation is:

537

$$
\nabla .\left(2\left[\frac{k}{\mu}\right] \frac{\rho \nabla p}{A}\right)=0
$$

$$
A=1+\sqrt{1+4 \rho \beta\left(\frac{k}{\mu}\right)^{2}|\nabla p|}
$$

where $p, \mu, k, \rho$ and $\beta$ are pressure, viscosity, absolute permeability, density and single phase inertial factor. 
As noted by (Jamiolahmady et al., 2006) the term A is obtained by relating the absolute velocity term $|\mathrm{v}|$ to the pressure gradient by solving the second order polynomial (Forchheimer, 1914a) equation for positive $|\mathrm{v}|$ in the case of a single phase linear Darcy flow system, A is equal to one.

The equations describing the two phase flow of gas and condensate around a horizontal well are:

$$
\nabla .\left(\left\{\left[\frac{\rho k_{r}}{\mu}\right]_{g}+\left[\frac{\rho k_{r}}{\mu}\right]_{c}\right\} k \nabla P\right)=0 .
$$

The total fluid composition $\left(\mathrm{z}_{\mathrm{j}}\right)$ is constant as the fluid flows through the porous media. However, for each component, there is mass transfer between two phases as expressed by the following equation:

$$
z_{j}=\frac{\rho_{g} y_{j} G T R+\rho_{c} x_{j}(1-G T R)}{\rho_{g} G T R+\rho_{c}(1-G T R)}=\text { cons. }
$$

where GTR is the total gas fraction ratio defined by Equation A-4.

$$
G T R=\frac{Q_{g}}{Q_{g}+Q_{c}}=\frac{1}{1+\frac{k_{r g}}{k_{r c}} \times \frac{\mu_{c}}{\mu_{g}}} .
$$

where $Q$ is the volumetric flow rate and $\mathrm{g}$ and c refer to gas and condensate.

In Equation A-2, relative permeability which varies with interfacial tension (i.e. pressure for a given fluid composition) and velocity is estimated using the correlation by (Jamiolahmady et al., 2009). In this correlation, gas relative permeability is interpolated between a base curve and a miscible curve, both corrected for the effect of inertia, using a generalised interpolation function. The correlation has either universal parameters or those parameters that can be estimated from readily available petrophysical data. The correlation is based on the relative permeability ratio $\left(\mathrm{k}_{\mathrm{rgtr}}=\mathrm{k}_{\mathrm{rg}} / \mathrm{k}_{\mathrm{rg}}+\mathrm{k}_{\mathrm{rc}}\right)$ as the main variable, which is closely related to fractional flow. The condensate relative permeability is calculated using the definition of relative permeability ratio. It should be noted that in gas/condensate systems, fractional flow is directly related to fluid composition and pressure at steady-state, which is generally prevailing near the wellbore, hence making it much more attractive practically, compared to saturation, which depends on core characteristics.

\section{Mathematical Solution Technique}

The governing non-linear partial differential equation (PDE), Equations A-1 and A-2 for single phase and two phase flow, is solved using Comsol multi-physic software (version 3.4, 2007), which uses the finite element method. The main dependent variable in this equation is pressure (p).

The boundary conditions applied to this system are:

1) Infinite conductivity for the wellbore (the pressure gradient in the wellbore has been ignored),

2) The pressure at outer boundary (external radius) is known,

3) The pressure at the inner boundary (wellbore radius) is known.

For the two phase flow of gas condensate the equations are solved for both $\mathrm{p}$ and GTR. The boundary conditions applied to the two phase system are those used for the single phase mathematical model as well as 
571

572

573

574

575

576

577

578

579

580

581 582

583

584

585

586

587

588

589

590

591

592

593

594

595

implying the constant total composition across the two phase region, so either the GTR or the total fluid composition is known.

\section{Appendix C-Numerical Solution for Two Phase Flow}

Numerical Solution for an Equivalent Open Hole Model for Two Phase Flow of Gas

Condensate:

\section{Governing Equations}

For the radial model, a combination of continuity and non-Darcy flow equations (Equation A-

4) can be written as follows:

$\nabla \cdot\left[\frac{1}{r}\left[\frac{k}{\mu}\right] \cdot\left[\left(\frac{\rho k_{r}}{\mu}\right)_{g}+\left(\frac{\rho k_{r}}{\mu}\right)_{c}\right] \cdot \frac{\partial p}{\partial r}\right]=0$.

The differential equation of above equation (B-1) for steady state pressure distribution is:

$$
\begin{aligned}
& \frac{r_{i+1 / 2}}{\Delta r^{2}} \times\left(\left(\left(\frac{\rho k_{r}}{\mu}\right)_{g}+\left(\frac{\rho k_{r}}{\mu}\right)_{c}\right) \times \frac{k}{\mu}\right)_{i+1} \times P_{i+1}-\left(\frac{r_{i+1 / 2}+r_{i-1 / 2}}{\Delta r^{2}}\right) \times\left(\left(\left(\frac{\rho k_{r}}{\mu}\right)_{g}+\left(\frac{\rho k_{r}}{\mu}\right)_{c}\right) \times \frac{k}{\mu}\right)_{i} \times P_{i}+ \\
& \frac{r_{i-1 / 2}}{\Delta r^{2}} \times\left(\left(\left(\frac{\rho k_{r}}{\mu}\right)_{g}+\left(\frac{\rho k_{r}}{\mu}\right)_{c}\right) \times \frac{k}{\mu}\right)_{i-1} \times P_{i-1}=0
\end{aligned}
$$

$$
(\mathrm{B}-2)
$$

$z_{j}=\frac{\rho_{g} y_{j} G T R+\rho_{c} x_{j}(1-G T R)}{\rho_{g} G T R+\rho_{c}(1-G T R)}=$ cons

For steady state conditions:

$$
\mathrm{P}_{1}=\mathrm{Pw}
$$

$\mathrm{P}_{\mathrm{n}}=$ Pres

where subscripts $\mathrm{w}$ and res refer to wellbore and external radius.

\section{Appendix D-Iterative Procedure for Effective Wellbore Radius Estimation}

As noted in the text, the effective wellbore radius formulations depend on the velocity, IFT and fluid properties. Thus, the calculation of the effective wellbore radius needs an iterative procedure, as described below:

1) First the effective wellbore radius is estimated based on the geometric skin:

$$
r_{w}^{\prime}=r_{w} \times e^{-S g} .
$$


It should be noted that Sg is the geometric skin for the Darcy flow regime and can be easily calculated using any available horizontal well productivity formulation i.e. Joshi, Borosiv, Economides, Goode, P. and Kuchuk, Babu, D. and Odeh, etc.

2) Based on the effective well bore radius, the pressure profile and flow rate are calculated using Equation 3. It should be noticed that the pseudo pressure calculation is a function of relative permeability or in other words of velocity; therefore an iterative procedure is required to estimate the pressure profile and the mass flow rate.

3) Using Equation 11 or 18, the effective wellbore radius is calculated.

4) If the difference between the new effective open hole wellbore radius calculated and the previous one is not negligible, the calculation is repeated from step 2 , otherwise the calculated effective well bore radius and mass flow rate are reported.

607 